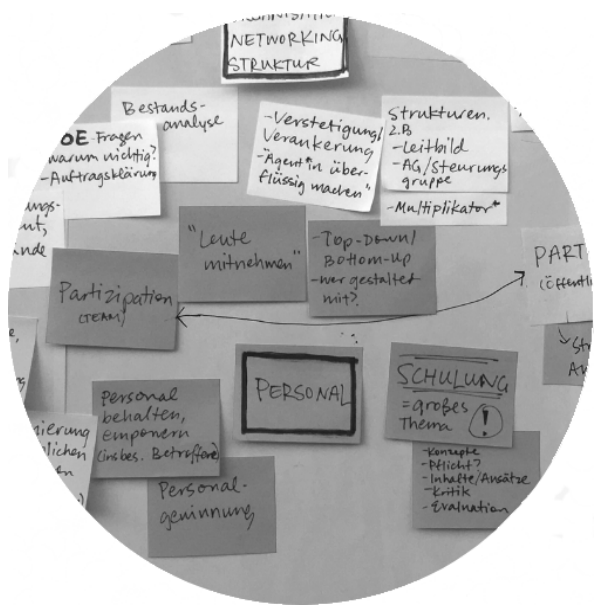

Teil II: Ran an die Strukturen! 



\section{Diversitätsorientierte öffnung als Prozess der Organisationsentwicklung in Bibliotheken}

Diversitätsorientierte Öffnung ist ein Querschnittsthema und ein Prozess der Organisationsentwicklung. Die Begleitung eines solchen Prozesses beinhaltet einige Aspekte, auf die in diesem Kapitel eingegangen wird: Dazu gehört die zirkuläre Auftragsklärung genauso wie der Umgang mit Widerständen und die Verankerung in den Strukturen der Institution. Um Anknüpfungspunkte zu bieten, werden übertragbare Instrumente und praktische Beispiele gegeben. Das Instrument der Arbeitsgruppe, das eine besondere Bedeutung in diesen Prozessen hat, wird am Ende des Kapitels anhand der Beispiele der Stadtbibliotheken Bremen und Heilbronn genauer vorgestellt.

Dieses Kapitel richtet sich gleichermaßen an Bibliotheksleitungen und Bibliotheksmitarbeitende, die sich mit Diversitätsorientierter Öffnung beschäftigen. Im Text werden daher im Wechsel beide Perspektiven eingenommen.

Die Autorinnen dieses Artikels sind im Rahmen von $360^{\circ}$ - Fonds für Kulturen der neuen Stadtgesellschaft ${ }^{1}$ im Bereich der diversitätsorientierten Organisationsentwicklung in Bibliotheken tätig.

Denise Farag ist in der Stadtbibliothek Heilbronn für Interkulturelle Bibliotheksarbeit und Diversität zuständig. Die Stadtbibliothek Heilbronn ist dem Schul-, Kultur- und Sportamt der Stadt Heilbronn zugeordnet. In der Zentralbibliothek, den zwei Stadtteilbibliotheken und einer Fahrbibliothek sind 35 Mitarbeitende beschäftigt.

Ruth Hartmann ist als Projektmanagerin für Interkulturelle Bibliotheksarbeit und Diversity an der Stadtbibliothek Bremen tätig. Die Stadtbibliothek Bremen ist als Eigenbetrieb dem Senator für Kultur der Freien Hansestadt Bremen zugeordnet, hat jedoch eine gewisse Eigenständigkeit im betrieblichen Wirtschaften. Zurzeit sind in der Zentralbibliothek, den sieben Zweigstellen und der Busbibliothek 156 Mitarbeitende ${ }^{2}$ angestellt.

Die Autorinnen haben sich für diesen Artikel dazu entschieden, den Begriff der Beraterin für die Person zu wählen, die den Prozess der Organisationsentwicklung begleitet. Damit soll ausgedrückt werden, dass diese Person nicht die-

1 Siehe dazu auch die Einleitung dieses Sammelbandes.

2 Stand 31.12.2020, inkl. der Auszubildenden, FSJlerinnen, Werksstudierenden etc.

ə Open Access. (C) 2021 Denise Farag \& Ruth Hartmann, publiziert von De Gruyter. (๕)BY Dieses Werk ist lizenziert unter der Creative Commons Attribution 4.0 Lizenz. 
jenige ist, die allein für die operative Umsetzung zuständig ist, sondern vorrangig der Bibliothek mit fachlicher Expertise zur Seite steht.

In diesem Kapitel wird ausschließlich das generische Femininum verwendet; damit wird auch die aktuelle Arbeitsrealität in den meisten Bibliotheken abgebildet.

\section{Warum denn sofort Organisationsentwicklung - kann eine Bibliothek nicht „einfach so“ geöffnet werden?}

Bibliotheken haben sich in den letzten 20 Jahren verstärkt der interkulturellen Arbeit ${ }^{3}$ gewidmet. Hierbei haben sie aufgrund der Dringlichkeit der gesellschaftlichen Entwicklungen dieser Zeit reagiert und weniger strategisch vorausschauend und proaktiv agiert. Vielfach wuchs dennoch der Wunsch, die reaktiv entstandenen Angebote $\mathrm{zu}$ bündeln und konzeptionell in die Organisation einzubinden. Hierfür haben Bibliotheken unterschiedliche Wege gewählt, zum Beispiel die Einrichtung oder Neudefinition der Position von Zielgruppenbeauftragten ${ }^{4}$ wie auch die Stärkung von Ehrenamtskreisen, die die Verantwortung für entsprechende Veranstaltungen wie etwa Sprachcafés übernahmen. Wieder andere Bibliotheken bewarben sich auf die $360^{\circ}$-Förderung mit dem Ziel der strategischen Integration des Themas in ihre Organisation ${ }^{5}$.

Sich als Kultureinrichtung, und in diesem Fall als Bibliothek, diversitätsorientiert zu öffnen, geht über die interkulturelle Arbeit hinaus. Wie in der Einführung dieses Sammelbandes dargelegt, drückt sich Diversität in den Dimensionen ethnische Herkunft, soziale Herkunft, Bildung, Weltanschauung, Geschlecht, Alter und geistige und physische Fähigkeiten aus. Zudem wird Diversität als intersektional betrachtet. ${ }^{6}$

Als Organisation das Thema Diversität anzugehen, also unter anderem gesellschaftliche Vielfalt abzubilden und Chancengleichheit zu bieten (Regionale Arbeitsstellen für Bildung, Integration und Demokratie [RAA] e. V., 2017, S. 1),

3 Wobei dieses Thema auch schon vorher präsent war, so z. B. durch das IFLA Multicultural Library Manifesto, das 2006 entwickelt wurde (International Federation of Library Associations and Institutions [IFLA], 2006).

4 In der Stadtbibliothek Bremen sind diese Positionen seit der Jahrtausendwende sukzessive für die Bereiche Erwachsene und Kinder eingerichtet worden.

5 Siehe auch das Vorwort des Sammelbandes.

6 Siehe auch die Einführung des Sammelbandes. 
bedeutet immer auch, einen Veränderungsprozess zu wagen. Dieser betrachtet die Bibliothek als Ganzes mit dem Ziel, gesellschaftliche Diversität nicht nur in einzelnen Bereichen, beispielsweise im Programm, unter den Besuchenden oder innerhalb der Belegschaft, sondern sie in der gesamten Struktur der Organisation zu verankern.

Es wird in diesem Artikel immer wieder von „Organisation“ gesprochen, was wird eigentlich darunter genau verstanden? Die Autorinnen nehmen in diesem Kapitel eine systemische Sichtweise ein. Aus dieser Perspektive ist eine Organisation ein System, bestehend aus Subsystemen, die miteinander kommunizieren und Beziehungen aufbauen. Diese wachsen, entwickeln sich und existieren „in der Spannung zwischen Dauerhaftigkeit und Vergänglichkeit“ (Königswieser \& Hillebrand, 2019, S. 30). Organisationen erfüllen außerdem einen Sinn und dienen den Mitgliedern des Systems zu einem Zweck (Königswieser \& Hillebrand, 2019, S. 30).

Eine Bibliothek ist aus diesem Verständnis heraus also ein System, welches verschiedenste Untergliederungen aufweist. Dazu gehören z. B. die unterschiedlichen Teams, die sich wiederum in kleinere Systeme, bis zu den einzelnen Mitarbeitenden hin, aufsplitten und in einer permanenten Wechselwirkung stehen. Der Sinn der Organisation, der Bibliothek als Ganzes, ergibt sich aus dem öffentlichen Auftrag, den diese hat. Für die einzelnen Mitarbeitenden kann durchaus ein anderer Sinn mehr Relevanz haben, z. B. Geld zu verdienen oder Sozialkontakte mit den Kolleginnen zu pflegen.

Was ist aus diesem Verständnis heraus dann Organisationsentwicklung? „Organisationsentwicklung ist eine Methode zur geplanten Veränderung größerer sozialer Systeme.“ (Grossmann et al., 2015, S. 9). In dieser Definition stecken die strategische Herangehensweise, der Blick auf die gesamte Institution und auf deren Wandel. Für einen Prozess der Organisationsentwicklung gibt es unterschiedliche Ansätze - sie können von der Hierarchiespitze (top-down) oder von den Mitarbeitenden (bottom-up) initiiert sein. Wie dies geschieht und wie die hierarchische Steuerung und die Kommunikation umgesetzt werden, beeinflusst die Akzeptanz der Veränderungen. Wenn versucht wird, Prozesse der Organisationsentwicklung durch Konsens zu steuern, so kann eine erhöhte sowie breitere Akzeptanz ermöglicht werden. Aus systemischer Sicht ist Partizipation der Belegschaft wünschenswert und hilfreich, da eine Verankerung in der Breite und damit eine Verstetigung der erzielten Veränderung nur erreicht werden kann, wenn sie vom System verinnerlicht wird (Königswieser \& Hillebrand, 2019, S. 30). Und da ein soziales System erheblich von Personen abhängig ist, sind es die Menschen, die Veränderungen anstoßen, sie entweder mit Energie umsetzen oder blockieren (König \& Volmer, 2018, S. 190). Dies unterstreicht die 
Bedeutung von Partizipation im Prozess der diversitätsorientierten Öffnung, welcher ohne diese nicht gelingen würde.

\section{Wie läuft Veränderung ab?}

Jeder Veränderungsprozess - und damit auch der einer diversitätsorientierten Öffnung - geht durch verschiedene Phasen (vgl. Abb. 4). Dabei handelt es sich um ein Schema, welches variabel ist und in vielen Fällen nicht linear verläuft: ${ }^{7}$

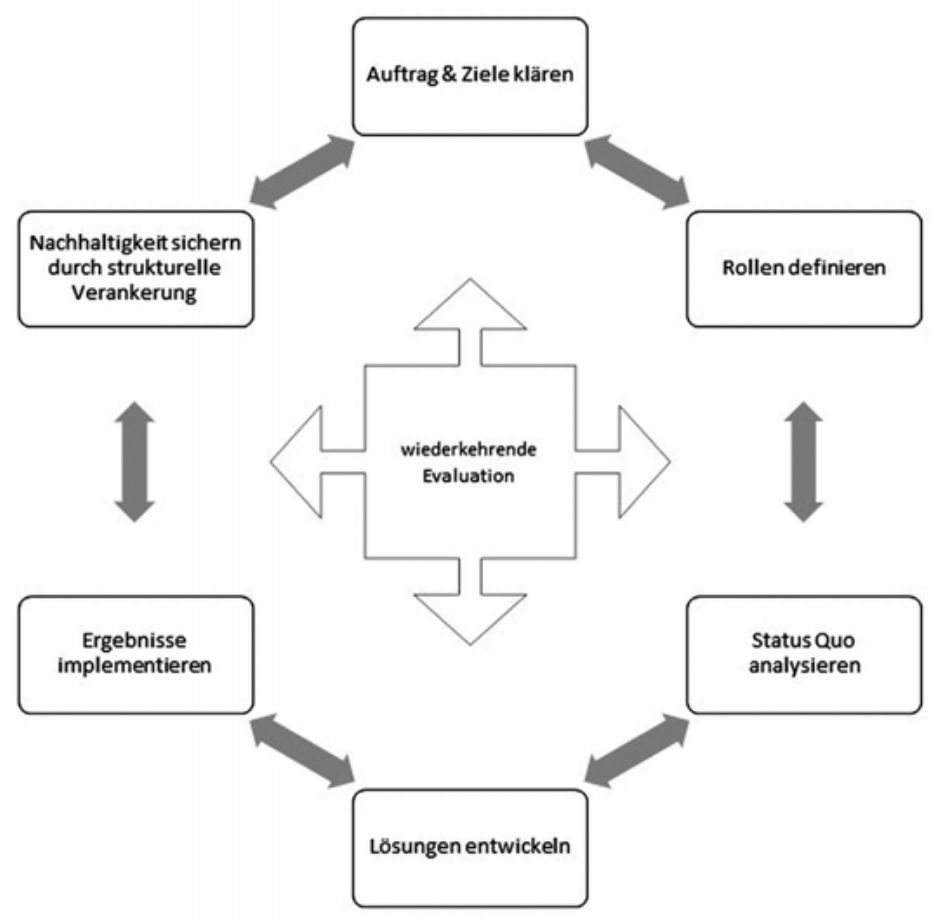

Abb. 4: Schritte im Veränderungsprozess. Ruth Hartmann (2020)

7 Das hier dargestellte Modell beruht auf den Erfahrungen der Autorinnen. In der Literatur gibt es verschiedene andere Ansätze wie z. B. das GROW-Modell, welches ähnliche Phasen definiert (König \& Volmer, 2018, S. 98 ff.). 
Genau dieser nicht-lineare Verlauf ist eine große Chance in Bezug auf den Prozess. Schritte werden kontinuierlich überprüft und evaluiert: Sind die Ergebnisse diejenigen, die erwartet wurden? Gibt es Entwicklungen, mit denen nicht gerechnet wurde? Gibt es Änderungen im Status quo, die bisher nicht berücksichtigt wurden? Permanent erfolgt die Rückkopplung zwischen Beraterin und Auftraggeberin, in diesem Kontext die Bibliotheksleitung. Dies kann sich als große Entlastung erweisen. Der variable Verlauf ermutigt alle Beteiligten kontinuierlich, die Ergebnisse zu reflektieren und lösungsorientiert anzupassen. Darüber hinaus schützt die wiederkehrende Feedback-Schleife davor, an den Zielen und Wünschen der Bibliotheksleitung vorbei zu agieren. Kurz gesagt: Die Leitung ist jederzeit informiert und kann ihre Gesamtverantwortung wahrnehmen.

Im Folgenden führen die Autorinnen durch die einzelnen Phasen eines Prozesses der diversitätsorientierten Öffnung. Der theoretische Hintergrund wird jeweils kurz dargestellt, auf den bibliothekarischen Kontext bezogen und nach Möglichkeit mit Beispielen verdeutlicht. Zur Veranschaulichung haben die Autorinnen eine fiktive Bibliothek gewählt. Diese Bibliothek hat das Ziel, den Prozess der diversitätsorientierten Öffnung voranzubringen und wird in diesem Kapitel dabei begleitet.

\section{Auftrag klären und Ziele formulieren}

Mit der Auftragsklärung steht und fällt der gesamte folgende Prozess. Dabei geht es nicht nur um inhaltliche Aspekte, sondern auch um die emotionale Ebene, also die Frage, ob zwischen der Bibliotheksleitung und der Beraterin eine konstruktive und vertrauensvolle Arbeitsbeziehung aufgebaut werden kann. In der systemischen Organisationsentwicklung gliedert sich die Auftragsklärung in vier Phasen, auf die hier nicht näher eingegangen werden kann. ${ }^{8}$ Wichtig ist aber, dass einige essenzielle Punkte berücksichtigt werden:

Zuallererst sollten die Ziele geklärt werden: Was genau wird von dem Prozess erwartet? Welche Ergebnisse möchte die Leitung erreichen?

Als sinnvoll kann empfohlen werden, erst einmal den IST-Zustand zu definieren. Was läuft gut, wo gibt es größeres Verbesserungspotenzial? Da dies manchmal nicht ganz einfach zu benennen ist, könnte es hilfreich sein, in einem ersten Schritt die Themenfelder festzuhalten. Im Fall Diversity hieße das, einmal alle Bereiche zu erfassen, in denen Diversity in der Bibliothek aktuell

8 Vgl. zu einer ausführlichen und gut verständlichen Beschreibung dazu König \& Volmer (2018, S. 93 ff.). 
eine Rolle spielt bzw. zukünftig spielen soll..$^{9}$ In einem zweiten Schritt kann dann die Leitung, z. B. anhand von skalierenden Fragen, den Status quo definieren und vergleichend betrachten.

Beispiel: Die Leitung der fiktiven Bibliothek definiert „Personalstruktur und internes Diskriminierungspotential“, „Kundinnen“ und „Netzwerkpartnerinnen“ als für sie wichtige Themenfelder. Nun lautet die Frage: „Auf einer Skala von 0-10, wobei Null für „nicht vorhanden“ und Zehn für „perfekt“ steht - wo sehen Sie den Bereich „Kundinnen“ aktuell in Bezug auf Diversität“?

Im nächsten Schritt schaut die Leitung, unterstützt von der Beraterin, in die Zukunft: Wo will sie mit ihrer Bibliothek hin? Geht es ihr um kleinere Anpassungen, „Wir möchten auf der Skala von einer Fünf auf eine Sechs“? Oder geht es ihr um „den großen Wurf“?

Tipp: Gerade, was Ziele angeht, tun sich viele Organisationen schwer. Es ist auch gar nicht so leicht, zu formulieren, wo man hinwill. Hier hilft es oft, mit paradoxen Fragen zu arbeiten: „Was dürfte auf keinen Fall passieren?“ oder aber auch „Was müsste die Bibliothek tun, um auf der Skala von einer aktuellen Fünf zukünftig auf eine Eins zu sinken?“

Seien Sie bei der Formulierung der Ziele mutig und bleiben Sie flexibel - so haben Sie später ausreichend Spielraum auch für kreative Lösungen, ohne von Ihren Zielvorgaben abweichen zu müssen (König \& Volmer, 2019, S. 81).

Darüber hinaus ist es bei der Formulierung von Zielen unbedingt zu empfehlen, das Kollegium mit einzubeziehen. Es geht nicht darum, im Verständnis einer Basisdemokratie gemeinsam darüber abzustimmen. Vielmehr ist durch eine hohe Transparenz zu gewährleisten, dass die Kolleginnen sich mitgenommen fühlen auf dem Weg. Niemand hat etwas von Plänen, die von der Belegschaft nicht mitgetragen werden. Eine Möglichkeit, die Beteiligung zu erhöhen, ist es, gemeinsam mit dem Kollegium die Ziele mit Meilensteinen zu unterlegen. Was genau heißt das von der Leitung definierte Ziel für meinen Bereich? Was kann mein Team dazu beitragen?

Zur Formulierung der Ziele - und der Meilensteine - gehört es auch, realistisch zu sein: Vorhaben, die vielleicht bei anderen Organisationen funktionieren, müssen nicht unbedingt die richtigen für Ihre Bibliothek sein. Oft formulieren Kolleginnen schon an diesem Punkt Vorbehalte - erste Widerstände tauchen auf. Nehmen Sie diese Vorbehalte ernst und gehen Sie konstruktiv mit ihnen um. ${ }^{10}$

9 Den Autorinnen ist bewusst, dass Diversity als Querschnittsthema gedacht in allen Bereichen eine Relevanz hat. Nichtsdestotrotz ist es gerade in der Anfangsphase sehr hilfreich, bestimmte Schwerpunkte zu setzen.

10 Siehe dazu auch „Veränderungen erzeugen Widerstand“ weiter hinten im Text. 


\section{Rollen definieren}

Aus Erfahrung der Autorinnen erweist es sich als sehr hilfreich, in diesem frühen Stadium die Rollen im Veränderungsprozess zu klären: „Rollen sind das personale Gegenüber einer Funktion.“ (Claus, 2016). Eine Rolle ist dementsprechend die auf die Person bezogene Erwartung, die das Gegenüber oder das System an eine Funktionsinhaberin richtet. Diese Rollen können vielfältig und durchaus auch gegensätzlich sein, je nachdem, aus welcher Perspektive und von wem auf die Funktionsinhaberin geschaut wird. Rollen können auch Selbstzuschreibungen sein.

Relativ einfach ist es, wenn die Beraterin von außen kommt. ${ }^{11}$ Hier sind Position, Funktion und Rolle in Bezug auf den Beratungsauftrag meist im Einklang. Herausfordernder wird es - und so ist es sehr oft der Fall -, wenn die Beraterin Teil der Institution selbst ist; ausgewählt vielleicht deshalb, weil sie ein besonderes Fachwissen mitbringt, welches hilfreich sein könnte.

Diese internen Beraterinnen befinden sich per se in einer ambivalenten Position und auch oft in einem Rollenkonflikt zwischen ihrem eigentlichen (Fach-) Auftrag und der nun neuen Rolle als Beraterin.

Beispiel: Die Leitung der fiktiven Bibliothek möchte gerne, dass Frau M., eine ihrer Teamleitungen, die Beratung in dem diversitätsorientierten Öffnungsprozess übernimmt. Frau M. ist in ihrem Team sehr beliebt und hat zudem noch Fachkenntnisse im Bereich Diversity. Jetzt soll sie u. a. einen genaueren Blick auf die Personalstruktur und auf mögliches Diskriminierungspotenzial werfen.

Können Sie sich das Dilemma vorstellen? Frau M. hat nun zwei Rollen: Teamleitung und Beraterin. Sie wird sich als Teamleitung mit Sicherheit immer schützend vor ihre Mitarbeitenden stellen wollen. Als Beraterin soll sie Diskriminierungspotenzial auch in ihrem Team aufdecken. Wo liegen jetzt die Loyalitäten? Bei der Bibliotheksleitung? Beim eigenen Team? Und wie sehen die Mitarbeiterinnen in ihrem Team die Beraterin? Haben sie Verständnis für die neue Rolle? Können sie die beiden Rollen voneinander trennen? Eine permanente Gratwanderung.

Eine solche Gratwanderung lässt sich für eine interne Beraterin nie ganz vermeiden. Es ist aber durchaus hilfreich, bestimmte Punkte schon im Vorfeld abzuklären, so z. B. die zeitlichen, finanziellen und ggf. auch personellen Ressourcen, die der Beraterin zur Verfügung stehen. Welche Entscheidungskompetenzen hat sie? Welche zusätzliche Unterstützung (z.B. durch Weiterbildung, Coaching, Supervision) benötigt sie? Organisationsentwicklung ist immer auf

11 Dies kann der Fall sein, wenn z. B. finanzielle Ressourcen die externe Beauftragung ermöglichen. 
zusätzliche Ressourcen dieser Art angewiesen und kann nicht „on top“ und nebenbei gemacht werden!

\section{Schriftliche Vereinbarung treffen}

Die ersten beiden Phasen der Auftragsklärung und der Definition der Rollen enden im Idealfall mit einer gemeinsamen Vereinbarung, die schriftlich festgehalten werden sollte: Oft laufen Prozesse über einen längeren Zeitraum und Vorgesetzte sowie Kolleginnen wechseln eventuell - da ist es für alle Beteiligten hilfreich, auch zu einem späteren Zeitpunkt noch einmal auf die Vereinbarung zurückgreifen zu können. Folgende Punkte sollten berücksichtigt werden (König \& Volmer, 2019, S. 38 ff.):

- Festlegung der Themen: In welchen Bereichen der Bibliothek sollen die Veränderungsprozesse stattfinden?

- Festlegung der Ziele ${ }^{12}$

- Festlegung von Indikatoren zur Überprüfung der Zielerreichung: Anhand welcher Aspekte kann festgestellt werden, dass die Organisation auf der Skala von Fünf auf Sechs gestiegen ist?

- Festlegung der Rahmenbedingungen: Neben der Rolle der Beraterin zählen hier auch Aspekte wie ein zeitlicher Rahmen, Ressourcen, Kommunikationswege und ähnliches.

\section{Status quo analysieren}

Um das System, das für den jeweiligen Veränderungsprozess relevant ist, analysieren zu können und sich gleichzeitig einen Überblick zu verschaffen, gibt es zahlreiche Instrumente, die hilfreich sein können. Die folgenden Methoden können von der Beraterin allein genutzt werden oder auch gemeinsam mit anderen Beteiligten. Die zweite Option bringt unterschiedliche Perspektiven zusammen, erhöht aber auch die Komplexität.

Die sogenannte Projekt-Umfeld-Analyse (PUMA) stellt das Projekt in den Mittelpunkt. Sie dient der Visualisierung der wichtigen Personen, Abteilungen, Partnerinnen, die auf die Gestaltung des Projektes oder Vorhabens Einfluss haben. Dies ermöglicht es, Beziehungen, Zusammenhänge und Ressourcen sichtbar zu machen und die Interessen und Erwartungen der Beteiligten in Bezug

12 Ziele können im Rahmen einer Schleife jederzeit wieder überprüft und angepasst werden. 
auf das Projekt zu verdeutlichen. Im nächsten Schritt können dann Strategien entwickelt werden, um diese Beziehungen im Sinne des Projektziels zu gestalten.

Ein Soziogramm fokussiert sich auf die sozialen Aspekte, verbildlicht die Beziehungen der beteiligten Personen noch genauer und stellt zudem die Kommunikation der Individuen in den Vordergrund. Es entsteht ein Diagramm aller Beteiligten; deren Nähe, Distanz und mögliche Konflikte werden eingezeichnet. Ein Blick auf dieses Diagramm erlaubt, ähnlich wie die PUMA, eine Sicht auf die Organisation, und es ergibt sich daraufhin die Möglichkeit, Aktionsschritte abzuleiten.

Egal, ob man sich für eine PUMA oder ein Soziogramm (vgl. Abb. 5) entscheidet - folgende Fragen können bei der Erstellung unterstützen:

- Wer ist beteiligt an dem Prozess?

- Wen betrifft die Veränderung auf welche Weise?

- Welche Erwartungen verbinden die Beteiligten mit dem Prozess und der Beraterin? Wer hat welche Interessen?

- Wer könnte vielleicht Sorgen oder Ängste mit dem Prozess verbinden?

- Welche Haltung haben die Beteiligten? Sind sie eher neugierig und zugewandt, eher abwartend oder reagieren sie ablehnend?

- Welche Auswirkungen könnten diese Haltungen möglicherweise auf den Prozess haben?

Nachdem der Blick auf die Organisation und die relevanten Akteurinnen hergestellt wurde, können anhand dessen Hypothesen für das weitere Vorgehen gebildet werden. Mit Hypothesen sind in diesem Fall Ideen für mögliche Handlungsschritte gemeint, die die Zielerreichung des Veränderungsprozesses unterstützen könnten.

Beispiel: Frau M. kann sich nun überlegen, wie sie die Kontakte zu den noch weiter entfernten Netzwerkpartnerinnen in der Stadt aufnehmen und aufbauen kann, oder, ob die Nähe zwischen Beraterin und Team der Bibliothek ausreicht und/oder wie das Team stärker in das Projekt eingebunden werden kann. Vielleicht auch, ob die Beziehung zu den anderen Führungskräften hinsichtlich des diversitätsorientierten Öffnungsprozesses direkter werden soll und wie dies gelingen könnte. $\mathrm{Zu}$ dem letzten Punkt stellt Frau M. die Hypothese auf, dass es hilfreich sein könnte, einen regelmäßigen Jour fixe mit den anderen Führungskräften einzuführen und diese dadurch verstärkt anzusprechen. 


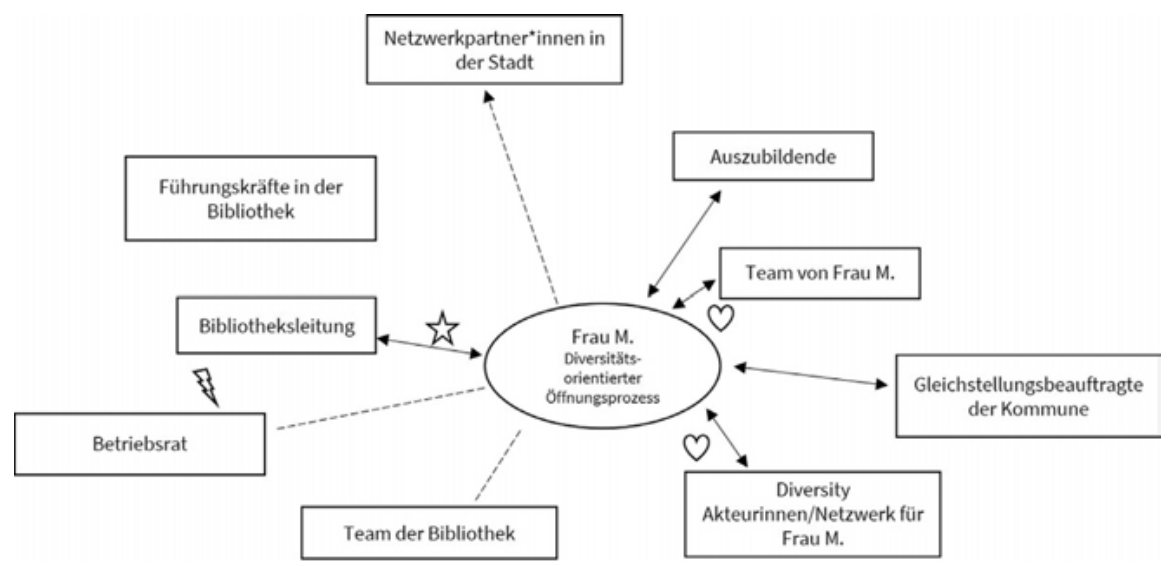

Abb. 5: Soziogramm. Denise Farag (2021)

Es bietet sich an, im Laufe des Prozesses immer wieder Soziogramme zu erstellen, um den erreichten Status quo mit der Ausgangssituation zu vergleichen.

\section{Passgenaue Lösungen entwickeln}

Es wurde im Abschnitt „Rollen definieren“ schon über die Rolle der Beraterin und der damit verbundenen Gratwanderung (Farag \& Hartmann, 2020, S. 54) gesprochen. Oft wird angenommen, dass es die Beraterin ist, die die Lösungen für das System - in diesem Falle die Bibliothek - findet. Die Rolle der Beraterin ist bei der Lösungsfindung jedoch eine andere: Sie steuert den Prozess, übernimmt das Prozesscontrolling und die Evaluation und kann auch an der Umsetzung beteiligt sein (Grossmann et al., 2015, S. 88). Wessen Aufgabe ist es denn dann, Lösungen zu finden? Die Antwort ist nicht erstaunlich: Das System, also die Bibliothek, sollte die Lösungen selbst finden. Es sind die Führungskräfte, die die Ziele und oft auch das Vorgehen vorgeben. Sie sind es, die ausreichende Ressourcen und die notwendigen Rahmenbedingungen sicherstellen (Grossmann et al., 2015, S. 85). Aber es sind die Mitarbeitenden, also das gesamte System, die an passgenauen Lösungen arbeiten. Dabei geht es weniger darum, wirklich mit dem gesamten Kollegium zu arbeiten. Die Autorinnen haben gute Erfahrungen mit Workshops oder auch Arbeitsgruppen (AGs) gemacht, die mit verschiedenen Ansätzen an die Lösungsfindung herangehen.

Beispiel: Eines der Ziele der fiktiven Bibliothek ist es, Kundinnen der verschiedenen Communities der Stadtgesellschaft noch gezielter anzusprechen 
und auch Zielgruppen zu erreichen, die bisher im Publikum unterrepräsentiert sind. Frau M. organisiert dazu einen Workshop. Zu dem Workshop lädt sie Kolleginnen aus allen Bereichen der Bibliothek, auch aus dem Kundenservice und der Öffentlichkeitsarbeit ein. Sie bringen weitere Sichtweisen und praktische Erfahrung mit ein.

Frau M. kann in einem Workshop oder einer AG die Vielfalt des Kollegiums abbilden. Aber in dem Beispielfall ist es darüber hinaus sehr $\mathrm{zu}$ empfehlen, auch die Expertise von außen einzubeziehen. Frau M. veranstaltet daher einen weiteren Workshop zu derselben Fragestellung, gemeinsam mit Vertreterinnen verschiedener Communitys. So bekommen die externen Perspektiven und wichtiger noch, die Perspektiven der Personen, die potenziell angesprochen werden sollen, Raum und können direkt berücksichtigt werden.

Weitere Möglichkeiten zur Partizipation externer Expertinnen sind, aus Sicht der Autorinnen, unter anderem externe Arbeitsgruppen oder Gremien wie beispielsweise ein Integrationsbeirat der Stadt, ein Freundeskreis der Bibliothek oder auch ein extra gegründetes beratendes Diversity-Komitee.

\section{Schritte zur Implementierung}

Nun, da der Status quo visualisiert wurde, gemeinsam Ziele definiert und erste Lösungsansätze in Form von Projekten und Maßnahmen erarbeitet wurden, heißt es für das gesamte System, sich auf genau diese ersten - vielleicht auch kleinen - Schritte im Veränderungsprozess einzulassen. Dafür ist eine transparente und in alle Hierarchieebenen laufende Kommunikation unerlässlich. Die Kolleginnen sollen wissen, was, warum, wie und wann geplant ist. Eine Möglichkeit dafür ist z. B. ein offizielles Kick-off-Treffen oder kontinuierliche StatusBerichte über das Intranet.

Beispiel: An dem oben genannten Workshop nahmen auch Vertreterinnen aus der LSBTIQ*-Community teil und bestätigten, dass sie sich zu wenig angesprochen fühlen. Aus diesem Grund hat Frau M. mit ihrer Leitung vereinbart, in einem ersten Schritt die Website und sämtliche Flyer der Bibliothek in Hinblick auf geschlechterumfassende Sprache zu überarbeiten. Sie bildete schon im Vorfeld eine Arbeitsgruppe mit Kolleginnen und externen Expertinnen, deren Mitglieder sich gemeinsam mit der Thematik beschäftigen. Noch bevor die neuen Sprachregelungen umgesetzt werden, holt sie das gesamte Kollegium ab durch Informationen im Intranet dazu, was geschlechterumfassende Sprache eigentlich ist und warum sie für die Bibliothek jetzt eine höhere Priorität hat. 


\section{Veränderungen erzeugen Betroffenheit}

Veränderungen im Rahmen eines diversitätsorientierten Öffnungsprozesses umfassen, wie beschrieben, die gesamte Organisation. Die Beteiligten sind jedoch auf unterschiedliche Weise betroffen, denn Veränderungen rufen verschiedenste Emotionen hervor, die auf den Prozess Einfluss haben können. Ein Blick auf die sogenannte Veränderungskurve, die ursprünglich aus der Trauerarbeit kommt und vielfach in Veränderungsprozessen angewendet wird, kann zur Einordnung, wo sich die Organisation oder auch Einzelne hinsichtlich des Prozesses aktuell befinden, dienen. ${ }^{13}$

Der Ablauf ist oft ähnlich (vgl. Abb. 6): So entsteht zu Beginn Schock, dass Veränderung nötig ist. Diese wird dann häufig verneint und abgelehnt. Die Phase kann unterschiedlich lang dauern, zumal in diversitätsorientierten Öffnungsprozessen der Impuls oft von außen kommt und die Dringlichkeit nicht aus dem System heraus entsteht. In der fiktiven Bibliothek war es z. B. die LSBTIQ*-Community, die den Anstoß zu einer Veränderung gab, welche in der Bibliothek selbst gar nicht als dringlich empfunden wurde.

In der Phase der rationalen Einsicht entsteht das Bewusstsein, dass sich etwas ändern muss. Das Tal der Tränen bringt dann die emotionale Akzeptanz mit sich, geht aber auch mit Trauer einher. Danach entstehen eine gewisse Energie und der Wille, Neues auszuprobieren. In der Phase des Ausprobierens liegt eine große Chance und zwar die, durch erfolgreiche Ergebnisse die Erkenntnis herbeizuführen, dass die Veränderung notwendig und gut ist. Die Phase der Integration bedeutet dann, dass die neue Aufgabe oder der neue Ablauf angenommen ist.

13 Die Veränderungskurve basiert auf den Fünf Phasen des Sterbens von Elisabeth KüblerRoss, wobei es hier um die geistige Verarbeitung des Abschieds vom Leben geht. Diese Kurve wurde dann auf die Reaktion von Beteiligten in Veränderungsprozessen übertragen (Grossmann et al., 2015, S. 113). 


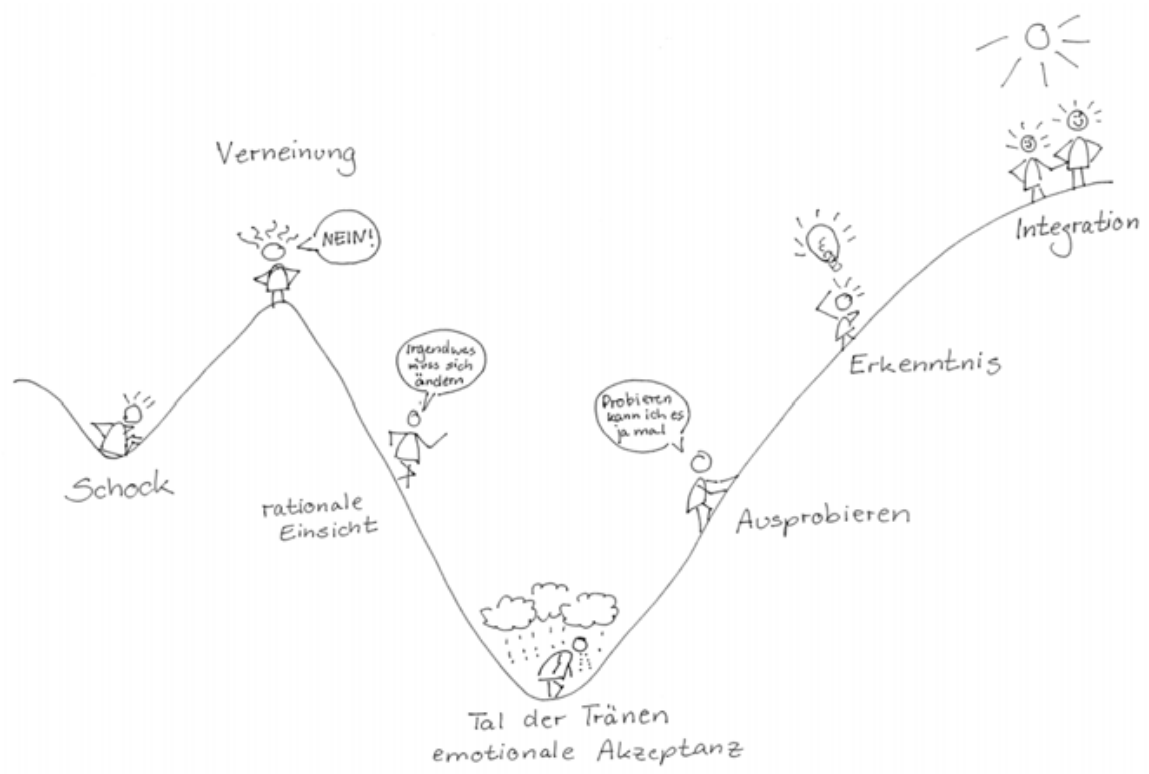

Abb. 6: Veränderungskurve. Ruth Hartmann (2020)

\section{Veränderungen erzeugen Widerstand}

Phasen wie Schock oder Verneinung gehen in der Regel mit Widerstand einher. „Von Widerstand kann immer dann gesprochen werden, wenn vorgesehene Entscheidungen oder getroffene Maßnahmen, die auch bei sorgfältiger Prüfung als sinnvoll, ,logisch“ oder sogar dringend notwendig erscheinen, aus zunächst nicht ersichtlichen Gründen bei einzelnen Individuen, bei einzelnen Gruppen oder bei der ganzen Belegschaft auf diffuse Ablehnung stoßen, nicht unmittelbar nachvollziehbare Bedenken erzeugen oder durch passives Verhalten unterlaufen werden." (Heins, o. J., S. 5). Stellen Sie sich dabei das Märchen des Rumpelstilzchens vor, welches mit hochrotem Kopf wütend auf den Boden stampft und immer wieder „Ich will nicht!“ brüllt. Allerdings ist dieser offen geäußerte Widerstand noch in der Hinsicht hilfreich, in der er erkennbar ist. Schwieriger wird es mit dem passiven Widerstand, der eben nicht sofort offensichtlich ist und sich durch Ausweichen oder Lustlosigkeit ausdrückt (Heins, o. J., S. 5).

Wie aber mit Widerstand umgehen? Hierbei helfen Prämissen der systemischen Haltung (Grossmann et al., 2015, S. 98 ff.): 
Das Bestehende sollte wertgeschätzt werden. Stellen Sie sich vor, Sie haben als Mitarbeiterin lange und intensiv daran gearbeitet, einen gewissen Status quo in Ihrer Bibliothek zu erreichen. Ihr Herzblut hängt daran! Jetzt kommt jemand und sagt, der Status quo ist schlecht und muss geändert werden. In der systemischen Arbeit gibt es kein Gut oder Schlecht. Es gibt ein Hilfreich und Weniger hilfreich. Soll heißen: Das Bestehende ist nicht schlecht. Es ist einfach im Hinblick auf eine sich ändernde Umwelt weniger hilfreich, als es vor einiger Zeit noch war.

Der Prozess sollte ergebnisoffen sein. Ja, die Führungskräfte formulieren die Ziele des Prozesses. Aber gleichzeitig ist es mehr als förderlich, die Wünsche, Bedürfnisse und Expertisen der Mitarbeiterinnen zu berücksichtigen. Und nein, es geht nicht um die Einführung einer Basisdemokratie à la „Die Mehrheit gewinnt“. Es geht vielmehr um ein ehrliches und wertschätzendes Ernstnehmen. ${ }^{14}$

Es braucht Mut zur Auseinandersetzung und zum Zulassen von Emotionen. Das ist vielleicht der wichtigste Punkt beim Umgang mit Widerständen. Auch wenn Vorbehalte aus der Belegschaft vielleicht unlogisch erscheinen - es ist wichtig, diese trotzdem ernst zu nehmen. Nur weil Sie die Emotionen der Kolleginnen nicht teilen, heißt das nicht, dass diese irrelevant sind. Geben Sie Raum, ermöglichen Sie es, diese Gefühle und Ängste zu formulieren: „Mitarbeitende erwarten nicht, dass Führungskräfte in ihrem Sinne entscheiden. Sie können auch anderslautende Entscheidungen akzeptieren. Was sie aber nur schwer akzeptieren können ist, dass ihre Meinung überhaupt nicht gehört wird, dass sie ihre Emotionen in Bezug auf die geplante Veränderung nicht zum Ausdruck bringen dürfen oder dass Argumente und Emotionen nicht ernst genommen werden." (Grossmann et al., 2015, S. 102).

Auf Basis dieser Grundannahmen erscheint der von Heins (o. J., S. 10) aufgezeigte Weg als überaus passend: Es geht nicht darum, Macht auszuüben und damit Widerstände $\mathrm{zu}$ ersticken, sondern es geht darum, Macht zu teilen und damit Widerstände einzubinden, diese $\mathrm{zu}$ verhandeln und zukünftig durch frühzeitiges Involvieren zu verhindern oder zu verringern.

14 Siehe hierzu auch den Abschnitt „Auftrag klären und Ziele formulieren“ in diesem Artikel. 


\section{Diversität als Querschnittsthema strukturell verankern oder: Wie macht die Beraterin sich überflüssig?}

Wenn es um einen Öffnungsprozess im Bereich Diversität geht, dann ist neben den vorab geschilderten Schritten besonders wichtig, dass die Entstehung einer „Diversity Bubble“ vermieden wird. Eine solche Blase entsteht, wenn das Thema Diversität in seiner Gänze bei einer Person angesiedelt wird. Von Übersetzungen für Flyer in Fremd- und Einfache Sprache, über geschlechterumfassende Formulierungen in Briefen, wofür die Hinterlegung im IT-System notwendig ist, bis hin $\mathrm{zu}$ den Führungen für Deutschkurse und Veranstaltungen $\mathrm{zu}$ LSBTIQ $^{\star}$ und Rassismus: Eine Person kann der Vielfalt der Themen, besonders aber deren Verankerung in der Gesamtorganisation, auf diese Art schwer gerecht werden. In diesem Zusammenhang sei das Beispiel der Frauenbeauftragten oder Inklusionsbeauftragten genannt: Hier werden oft Personen, die bereits in einer Institution tätig sind, mit der jeweiligen Zusatzaufgabe betraut. Nicht nur das „on top“ ist dabei problematisch, sondern auch die Tatsache, dass nun alles im Weitesten zum Thema passende an diese Person abgegeben wird. Der Rest der Institution setzt sich kaum weiter damit auseinander.

Das heißt: Diversitätsorientierte Öffnung benötigt Beteiligung. Möglichst viele aus dem Team der Bibliothek sollen am Öffnungsprozess mitwirken und ihre Perspektive einbringen. Aber nicht nur das - bei einem diversitätsorientierten Öffnungsprozess geht es vielfach um Dinge, die innerhalb der Institution noch nicht bekannt sind: Welche Bedürfnisse und Interessen haben die heterogenen Zielgruppen der Stadtgesellschaft? Welche Bedürfnisse hat eine heterogene Belegschaft? Passend zum Beispiel von Frau M. ist Teil des Prozesses also, gemeinsam festzustellen, welche Perspektiven von innen eingenommen werden können und welche nicht, weil sie schlicht im Kollegium bisher fehlen. Um die Bibliothek aus allen unterschiedlichen Blickwinkeln betrachten zu können, ist es folglich wichtig, die betreffenden Kolleginnen und Akteurinnen in der Stadtgesellschaft zur Beteiligung am Öffnungsprozess einzuladen - wie es Frau M. auch getan hat. Hieran wird deutlich, wie relevant die Sensibilisierung für und das Bewusstsein über Perspektiven sind und welchen bedeutenden Stellenwert die Netzwerkarbeit in einem solchen Öffnungsprozess hat - und zwar nach innen und außen.

Strukturelle Verankerung in diesem Kontext bedeutet, dass Diversitätsorientierte Öffnung kein Projekt ist, sondern ein fortlaufender Prozess, der auch in engem Zusammenhang mit dem gesellschaftlichen Wandel steht. Die angespro- 
chene Beteiligung ist in diesem Prozess eben auch der Schlüssel zur Nachhaltigkeit. Denn, unabhängig davon, ob es eine extra Personalstelle gibt, die ausschließlich und unbefristet für den Öffnungsprozess zuständig ist und diesen auf Dauer vorantreibt, sollte sich die Auseinandersetzung mit dem sich stetig wandelnden und entwickelnden Feld nachhaltig innerhalb der Organisation weitertragen. Wie eingangs erwähnt, erweisen sich interne Arbeitsgruppen hierfür als besonders hilfreich.

Was bedeutet es nun, wenn sich die diversitätsorientierte Öffnung in der Bibliothek weiterträgt? Die Beraterin wird „überflüssig“. Dies geht Hand in Hand damit, dass die Auseinandersetzung mit Diversität in die Gesamtorganisation überführt wird. Diese Überführung bedeutet die Verinnerlichung, die Akzeptanz und die Wertschätzung von Diversity als Querschnittsthema. Die Institution, die Bibliothek als Ganzes, hat die Verantwortung für den Prozess und die Veränderung übernommen - bis zu dem Punkt, an dem Impulse von innerhalb oder außerhalb der Bibliothek die Leitung wiederum zu der Erkenntnis bringen, dass ein Veränderungsprozess erneut angestoßen werden soll. Es schließt sich der Kreis - Veränderung ist wiederkehrend.

\section{AG Diversity \& Diversity Labor}

Im Folgenden werden die AGs der Stadtbibliotheken Bremen und Heilbronn vorgestellt. Das Beispiel der Bücherhallen Hamburg ist als Praxisbeispiel diesem Kapitel nachgestellt.

\section{AG Diversity in der Stadtbibliothek Bremen}

Wie kann Diversität langfristig als Querschnittsthema etabliert werden? Aus Sicht der Autorin, Projektmanagerin für Interkulturelle Bibliotheksarbeit und Diversity in der Stadtbibliothek Bremen, geht dies nur durch strukturelle Verankerung. Nur so kann aus ihrer Perspektive garantiert werden, dass Diversität unabhängig von den Interessen einzelner und deren Engagement gelebt wird. Nur so kann das Thema auf viele Köpfe verteilt werden und auch einzufordern sein.

Aus diesem Grund initiierte die Autorin Ende 2019 die AG Diversity, die aus vier Kolleginnen besteht, welche Schlüsselpositionen im Haus innehaben. Alle vier Kolleginnen haben einen übergreifenden Wirkungsbereich und durch Stu- 
dium oder Weiterbildung ${ }^{15}$ erworbene vertiefte Kenntnisse im Bereich Diversity. Die AG ist bewusst klein gehalten, wird aber themenbezogen durch interne und externe Expertinnen ergänzt. Die Rolle der Autorin ist ausschließlich beratend und moderierend - die AG, als integraler Teil der Stadtbibliothek Bremen, ist Expertinnengremium für ihr eigenes System, die Bibliothek.

Der Blick der AG richtet sich auf die Bereiche Personal, Publikum \& Netzwerk und Programm. Ergänzt wird dieser Blick durch übergeordnete Fragestellungen nach Partizipation, Zugangshürden, Methodenkompetenz und Ressourcen. Ziele wurden in Hinblick auf diversitätsorientierte Öffnung formuliert und mit Meilensteinen unterlegt. Die Meilensteine wiederum werden in Bezug auf Verantwortlichkeiten, benötigte Ressourcen und Priorität im Prozess analysiert. Wichtig ist der AG, dass keine Parallelstrukturen geschaffen werden, dass also, wann immer möglich, auf Teams der Bibliothek oder bestehende themenspezifische AGs im Haus zurückgegriffen wird.

Beispiel: Eines der formulierten Ziele ist die Sichtbarmachung und bessere Repräsentanz möglichst aller Teile der Stadtgesellschaft in der Kommunikation und im Auftritt der Stadtbibliothek. Ein Meilenstein dazu ist die Erstellung einer Checkliste für Kommunikation und Marketing. Durch die Checkliste sollen Standards geschaffen werden, u. a. in Bezug auf zielgruppengerechte Sprache. Anhand einer RACI-Analyse ${ }^{16}$ verdeutlicht die AG die Zuständigkeiten: In diesem Fall sieht sie die Zuständigkeit nicht prioritär bei sich, sondern beim Team „Marketing und Kommunikation“. Ihre eigene Rolle definiert sie lediglich als initiierend und beratend; in der gleichen Rolle sieht sie die schon bestehende AG Interne Service- und Kommunikationsstandards. Durch die kontinuierliche Feedbackschleife mit der Betriebsleitung wird garantiert, dass diese hinter den Vorschlägen steht. In einem nächsten Schritt läge die Verantwortung bei der Leitung, dass sie die Abteilung „Marketing und Kommunikation“ mit der Erstellung dieser Checkliste beauftragt.

Auf diese Art und Weise können die Ziele in Hinblick auf diversitätsorientierte Öffnung möglichst ressourcenschonend in schon bestehende Strukturen integriert werden.

Ziel der AG Diversity ist es übrigens, sich auf längere Sicht selber überflüssig zu machen.

15 Vgl. dazu auch die Vorstellung der Diversity-Multiplikatorinnen im Kapitel „Diversitätsorientierte Personalgewinnung und -entwicklung“ der gleichen Autorin in diesem Sammelband. 16 RACI steht für responsible (verantwortlich), accountable (im Sinne von rechenschaftspflichtig, dies ist meist die Betriebsleitung), consulting (beratend), informed (informiert). Diese Analyse ist ein empfehlenswertes Tool, um sich einen Überblick zu schaffen, wer in einem Projekt / einer Maßnahme etc. eigentlich welche Rolle hat. 


\section{Diversity Labor der Stadtbibliothek Heilbronn}

Das Diversity Labor der Stadtbibliothek Heilbronn wurde zu Beginn des Jahres 2020 gegründet - eineinhalb Jahre nachdem sich die Bibliothek durch die $360^{\circ}$. Förderung auf den Weg der diversitätsorientierten Öffnung gemacht hat. In dieser Zeit wurde das ganze Kollegium für Diversität, den Prozess und die Veränderung sensibilisiert. Die Autorin gewann als Beraterin Einblicke in die Bibliothek, ihre Strukturen, bestehende Arbeitsgruppen und die Organisationskultur, die für die Gründung wertvoll waren.

So ergab sich als Ziel, für die Gründung und für den Charakter der „Arbeitsgruppe“, folgende vier Aspekte umzusetzen:

- Repräsentanz aller Fachbereiche der Bibliothek im Diversity Labor,

- Umsetzungsstärke und Verbindlichkeit,

- Mitgestalten,

- Gemeinsames Lernen.

Diese vier Merkmale wurden bei der Vorstellung in den Mittelpunkt gestellt. Alle Interessierten wurden zur Beteiligung eingeladen. Die Teilnahme ist freiwillig und auf diese Art gelingt es, in der Gruppe einen Querschnitt der Bi-bliothekshierarchien und -abteilungen, bestehend aus acht Personen, abzubilden. Über die Zusammenarbeit wird die Diversity-Arbeit sichtbarer, und die Teilnehmerinnen werden am Prozess der Öffnung stärker beteiligt. Die Umsetzungsstärke und Verbindlichkeit ist auch durch Entscheidungsmacht gegeben. Diese ist durch die Teilnahme der Hausleitung gewährleistet. In den monatlichen Sitzungen des Diversity Labors werden verschiedene Themen bearbeitet (von Publikumsveranstaltungen bis hin zur Frage: „Wie erreichen wir die Kolleginnen besser?"), immer mit der Absicht, dass sie auch durch das Labor in die Fachbereiche getragen werden und somit durch die Gruppe direkt zur Umsetzung gebracht werden können. Das gemeinsame Lernen bedeutet, dass sich Teilnehmerinnen durch Webinare oder Schulungen mehr Wissen zu Diversität aneignen.

Wichtig ist seit der Gründung, dass alle Themen, die bearbeitet werden und mögliche Veränderungen für die Bibliothek nach sich ziehen, mit dem Diversity Labor gemeinsam entstehen. Sollte der Impuls durch die $360^{\circ}$-Agentin kommen, wird er durch das Diversity Labor befürwortet und klar unterstützt. Das Diversity Labor setzt sich die Ziele selbst und soll auch über den Zeitraum der $360^{\circ}$-Förderung hinaus bestehen.

Ein Beispiel der schnellen und fruchtbaren Arbeit des Diversity Labors für die Stadtbibliothek Heilbronn sind die Montagsposts auf den Kanälen der Sozialen Medien. Seit Juni 2020 wird jeden Montag unter dem \#MontagGegenDiskriminierung ein Medientipp gepostet, der sich mit Diversität beschäftigt und auf 
einem Vorschlag aus dem Team der Bibliothek beruht. So wurden zum einen alle im Team aufgerufen, Literatur zu allen Diversitätsdimensionen zu entdecken und sich zu beteiligen, und zum anderen werden die Kundinnen der Bibliothek mit relevanten Tipps versorgt. Außerdem wird damit nach außen sichtbar, dass sich die Bibliothek mit Diversität kritisch auseinandersetzt, selbst dazulernt und sich im Prozess der Öffnung befindet.

Für den verbleibenden Förderzeitraum steht, neben der regulären Arbeit des Diversity Labors, die Vernetzung der Teilnehmerinnen mit relevanten Kooperationspartnerinnen in der Stadt im Fokus. Dies wird unter anderem umgesetzt durch die Einführung sogenannter Veranstaltungspatinnen des Diversity Labors. Alle Programmpunkte und Veranstaltungen sollen durch eine Veranstaltungspatin begleitet werden. Dazu gehört auch die Teilnahme an einem Austauschtermin mit den Kooperationspartnerinnen.

Zur regulären Arbeit gehört auch die wiederkehrende Evaluation des Status quo. Also die Fragen: „Wo stehen wir aktuell? Und wo möchten wir noch hin?“

Und der Kreis schließt sich. Das Diversity Labor und allgemein interne Diversity AGs können fortlaufend versuchen ${ }^{17}$, einen „kritischen“ Blick auf die Institution zu werfen und sich somit immer wieder neue Ziele zu setzen und diese weiterzuentwickeln.

\section{Literaturverweise}

Claus, P. (2016, 10. Februar). Führung aus der Perspektive von Position, Funktion und Rolle. Höhere Berufsbildung Uster (HBU): https://www.hbu.ch/de/Fuehrung_Position_Funkti on_Rolle.b61.10428.html\#: :text=Funktion\%20oder\%20Funktionsprofil\%20beschreibt\% 20den,gesamten\%200rganisation\%20zu\%20leisten\%20ist.\&text=Rolle(n)\%20sind\% 20das\%20personale\%20Gegen\%C3\%BCber\%20der\%20Funktion (6.7.2020).

Farag, D. \& Hartmann, R. (2020, Januar). Diversitätsorientierte Öffnung als Chance. (B. I. e. V., Hrsg.) BuB - Forum Bibliothek und Information (01/2020), S. 52-57.

Grossmann, R., Bauer, G. \& Scala, K. (2015). Einführung in die systemische Organisationsentwicklung. Heidelberg: Carl-Auer Verlag GmbH.

Heins, S. (o. J.). Widerstände in Veränderungsprozessen abbauen. Organisationsberatung Seminare - Coaching: http://www.sabine-heins.de/user_files/datei_1245233473_25388. pdf (17.12.2020).

International Federation of Library Associations and Institutions IFLA. (2006). The IFLA Multicultural Library Manifesto. The Multicultural Library - a gateway to a cultural diverse: https://archive.ifla.org/VII/s32/pub/MulticulturalLibraryManifesto.pdf (5.2.2021).

17 „Versuchen“ ist so zu verstehen, dass sich die Autorin bewusst ist, dass es hilfreich ist, den internen Blick unbedingt durch externe Perspektiven zu erweitern. Dafür sind die Vernetzung und ein beratendes, externes Diversity-Gremium weitere mögliche Maßnahmen. 
König, E. \& Volmer, G. (2018). Handbuch Systemische Organisationsberatung. Weinheim, Basel: Beltz.

König, E. \& Volmer, G. (2019). Handbuch Systemisches Coaching. Weinheim Basel: Beltz.

Königswieser, R. \& Hillebrand, M. (2019). Einführung in die systemische Organisationsberatung. Heidelberg: Carl-Auer Verlag $\mathrm{GmbH}$.

Regionale Arbeitsstellen für Bildung, Integration und Demokratie (RAA) e. V. (2017). Diversitätsorientierte Organisationsentwicklung: Grundsätze und Qualitätskriterien. https:// raa-berlin.de/wp-content/uploads/2018/12/RAA-BERLIN-DO-GRUNDSAETZE.pdf (9.2.2021). 